\title{
Durability Improvement of Synthetic Dry Adhesives by Metal Coatings
}

\author{
Gyuhe Kim, Taechang Ahn, and Hui Yun Hwang \\ Department of Mechanical Design Engineering, Andong National University, 1375 Gyeongdong-ro, Andong-si, \\ Gyeongsangbuk-do 760-749, Republic of Korea
}

Correspondence should be addressed to Hui Yun Hwang; hyhwang@andong.ac.kr

Received 17 May 2017; Revised 1 August 2017; Accepted 16 August 2017; Published 20 September 2017

Academic Editor: Mikhael Bechelany

Copyright (C) 2017 Gyuhe Kim et al. This is an open access article distributed under the Creative Commons Attribution License, which permits unrestricted use, distribution, and reproduction in any medium, provided the original work is properly cited.

Gecko-like synthetic dry adhesives (SDAs) have adhesion comparable to that of a real Gecko's foot, but with very low durability. To address this problem, self-cleaning or stiff core embedding methods have been proposed. However, the proposed methods require special locomotion or complicated manufacturing. In this study, we suggested a metal coating on synthetic dry adhesives to improve durability. SDAs were fabricated via PDMS. Then, metals such as indium, zinc, and gold were coated on the SDAs. The adhesion tests show that the indium- and zinc-coated microstructures have a higher shear adhesion strength than the noncoated ones. Also, the shear adhesion strength of noncoated SDAs was only $14.5 \%$ of the initial strength while that of the zinc-coated ones was $35.6 \%$ after 200 times of attachment and detachment. We could find PDMS debris and fractures on noncoated SDAs, which results in weakening of the adhesion strength. On the other hand, a relatively high hardness, strength, and stiffness of the zinc coating layers reduced the wear and fractures of the micropatterns, which led to an improved durability in the SDAs. From these tests, we can conclude that the metal coating method could improve the durability of the SDAs.

\section{Introduction}

Many researchers have engineered systems with a higher efficiency by adopting novel features that have been observed in nature. As such, synthetic dry adhesives (SDAs) inspired by Gecko's feet are a typical example of systems inspired by nature $[1,2]$. Gecko's feet are widely known to have millions of microsized setae with over 1,000 nanosized spatulae in hierarchical structures, and the Van de Waals force between the spatulae and foreign surfaces gives the superb adhesion to Gecko's feet. In addition, Gecko's feet have other superior features, including directionally different and reversible adhesion, self-cleaning, and nonpollution characteristics. Therefore, there have been many attempts to mimic Gecko's feet for industrial applications in, for example, medical patches and sticky robot hands [3-16].

In the initial phase (earlier stage), micro- or nanopatterned structures with a high aspect ratio were designed for SDAs, and their adhesion characteristics were evaluated using a pull-off test, friction test, and peeling test [17-19]. Tilted or hierarchical structures with $3 \mathrm{D}$ shaped-tips, such as with a mushroom shape, and self-cleaning properties were tested to improve the adhesion [20-27]. These researches were focused on how to fabricate SDAs with very small and complicated structure, and various microelectronic and micromechanical processes were applied. Dip transfer, multistep photolithography, UV-assisted CFL, and multistep template are typical examples of numerous trials. Micro- and nanopillars with a higher aspect ratio are essential to achieve reasonable adhesion, but the aforementioned fabrication methods cannot be used commercially since extremely precise equipment and a long time are required for fabrication [24, 28-41]. In contrast, polymers or elastomers such as polydimethylsiloxane (PDMS) have been widely used for SDAs due to their flexibility and ease of fabrication. However, these materials have a low stiffness and strength, which reduces the adhesion strength of the micro- and nanopillars due to fiber collapse, abrasion, and fracture [1, 22, 42-44]. This limits SDAs to only a single application, such as medical patches, whereas demand for applications for robot hands and transportation equipment for clean rooms requires a more durable adhesion. Therefore, maintaining the adhesion strength of the SDAs can 
TABLE 1: Specifications of polydimethylsiloxane (SYLGAD 184, Dow Corning Co. Ltd., USA).

\begin{tabular}{lcc}
\hline Property & Unit & Value \\
\hline & $\mathrm{cP}$ & 5175 \\
Viscosity (part A) & $\mathrm{mPa}-\mathrm{sec}$ & 5175 \\
& $\mathrm{~Pa}-\mathrm{sec}$ & 5.2 \\
\hline & $\mathrm{cP}$ & 3500 \\
Viscosity (mixed) & $\mathrm{mPa}-\mathrm{sec}$ & 3500 \\
& $\mathrm{~Pa}-\mathrm{sec}$ & 3.5 \\
\hline Specific gravity (cured) & - & 1.04 \\
\hline Working time at $25^{\circ} \mathrm{C}$ & $\mathrm{hr}$ & 1.4 \\
\hline Cure time at $25^{\circ} \mathrm{C}$ & $\mathrm{hrs}$ & 48 \\
\hline Heat cure time @ $100^{\circ} \mathrm{C}$ & $\mathrm{min}$ & 35 \\
\hline
\end{tabular}

increase their applicability to a wider range. One method that was proposed to maintain the adhesion strength was to coat it with an adhesive structure. Suh's group reported on the adhesion strength of nanohairy dry adhesives coated with a $4 \mathrm{~nm}$ thick platinum layer [22], and Kim's group also presented dry adhesives with micropatterns coated with a $6 \mathrm{~nm}$ thick gold layer [45]. However, these studies have only given the changes in the adhesion strength with and without metal coatings.

The purpose of this study is to investigate the changes not only of the adhesion strength, but also of the structural integrity by considering the mechanical and physical properties of the coating materials and their thickness. We fabricated SDAs with micropatterns using PDMS and coated three different metals on the micropatterns with different thicknesses via plasma sputtering. We then measured the adhesion strength of the fabricated dry adhesives during repeated shear tests on glass surfaces and observed the structural changes with an optical microscope as well as a scanning electron microscope (SEM). For further analysis, we compared the changes in the adhesion strength and structural integrity based on the material properties and thickness of the coating metals.

\section{Materials and Methods}

2.1. Materials and Specimen. Many studies have used polydimethylsiloxane (PDMS), polyurethane (PU), and polyurethane acrylate (PUA) to develop SDAs. We selected PDMS (SYLGARD 184, Dow Corning Co. Ltd., USA) due to its easy micro- and nanofabrication, relatively high durability, and flexibility. The specifications of the PDMS are shown in Table 1.

Gold, indium, and zinc were selected as coating materials because they have different stiffness, strength, and hardness, as shown in Table 2. These properties were expected to have an effect changes in the adhesion strength, durability, and structural integrity.

2.2. Sample Preparation. SDAs with micropatterns were fabricated using the process shown in Figure 1. The base material (SYLGARD 184 Base, Dow Corning Co. Ltd., USA) and curing agent (SYLGARD 184 Curing, Dow Corning Co. Ltd.,
TABLE 2: Specifications of cured PDMS and coating metals.

\begin{tabular}{lccccc}
\hline & $\begin{array}{c}\text { Purity } \\
(\%)\end{array}$ & $\begin{array}{c}\text { Strength } \\
(\mathrm{MPa})\end{array}$ & $\begin{array}{c}\text { Stiffness } \\
(\mathrm{GPa})\end{array}$ & $\begin{array}{c}\text { Hardness } \\
(\mathrm{HV})\end{array}$ & Company \\
\hline Indium & 99.99 & 4.5 & 12.7 & $<10$ & iNecus.lnc. \\
Zinc & 99.99 & 37 & 96.5 & 30 & iNecus.lnc. \\
Gold & 99.99 & 102 & 77 & 25 & iNecus.lnc. \\
\hline
\end{tabular}

USA) were mixed at a ratio of 10:0.8 using a planetary centrifugal mixer (ARM-310, Thinky Co., Japan) under a rotating speed of 2,000 rpm for $1 \mathrm{~min}$. Then, the mixture was poured in a micropatterning mold with microholes with a diameter of $30 \mu \mathrm{m}$, height of $60 \mu \mathrm{m}$, and interval of $30 \mu \mathrm{m}$. The mixtures were poured in the mold and cured at $80^{\circ} \mathrm{C}$ for 3 hours under vacuum in a vacuum oven (VO-64, Hanyang Scientific Equipment Co. Ltd., Korea). Finally, we could obtain SDAs with micropatterns once they were separated from the mold. Subsequently, micropatterns were coated with metal materials (gold, zinc, and indium) using a compact plasma sputtering coater (GSL-1100X-SPC12-LD, MTI Co., USA). DC argon plasma sputtering was conducted under gas purity of $99.995 \%$, gas pressure of $8 \mathrm{~Pa}$, discharge power of $2.4 \mathrm{~W}$, and the sputtering time ranging from $4 \mathrm{~s}$ to $20 \mathrm{~s}$ to control the coating thickness from $2 \mathrm{~nm}$ to $10 \mathrm{~nm}$.

2.3. Test Method. The macroscopic shear adhesion strength of the SDAs was measured using a frictional adhesion test, as shown in Figure 2 since the fabricated SDAs only had simple micropatterns $[29,46]$. Specimens with sizes of $10 \mathrm{~mm}$ $\times 10 \mathrm{~mm}$ were attached to a slide glass with a preload of $2 \mathrm{~N}$. A moving stage with a load cell at $30 \mathrm{~N}$ (DBCM, Bongshin Load Cell Co., Ltd., Korea) pulled off the specimens at the speed of $0.03 \mathrm{~mm} / \mathrm{s}$, and the frictional forces were recorded during the tests. Upon completing these tests, the thickness of each coating material with a maximum shear adhesion strength was selected. Frictional adhesion tests were then repeated 200 times with metal-coated SDAs with selected thickness, and the changes were analyzed regarding the shear adhesion strength to evaluate their durability.

In addition, we observed changes in the surfaces and structural integrity of micropatterns for every tenth frictional adhesion test using a digital microscope (KH-7700, Hirox Co., Ltd., Japan) and a field emission scanning electron microscope (JSM-6700F, Jeol Ltd., Japan).

\section{Results and Discussion}

3.1. Thickness Selection of the Coated Metal Layers. Figure 3 shows the measured shear adhesion strength of the metalcoated SDAs for each material and the thickness of the metal films on the adhesives. As reference, the SDAs without metal coatings had a shear adhesion strength of $9.1 \mathrm{kPa}$. The shear adhesion strength of the SDAs coated with indium increased from $3.9 \mathrm{kPa}$ to $10.9 \mathrm{kPa}$ when the indium thickness increased from $2 \mathrm{~nm}$ to $4 \mathrm{~nm}$, and the shear adhesion strength with an indium thickness ranging from $4 \mathrm{~nm}$ to $10 \mathrm{~nm}$ decreased from $10.9 \mathrm{kPa}$ to $4.51 \mathrm{kPa}$. The maximum adhesion strength was $10.9 \mathrm{kPa}$ for the $4 \mathrm{~nm}$ thick indium layer, and there was 


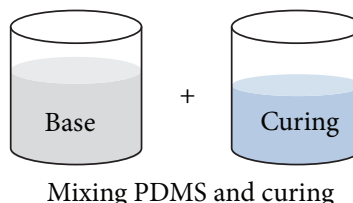

Mixing PDMS and curing agent (mixing ratio of $10: 0.8$ )
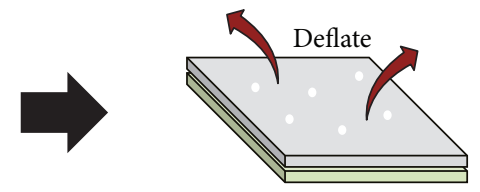

Deflate the bubbles in the mixture (vacuum gage $-0.07 \mathrm{Mpa} / 30 \mathrm{~min}$ )
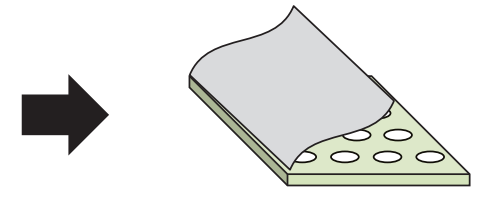

Cooling and separating

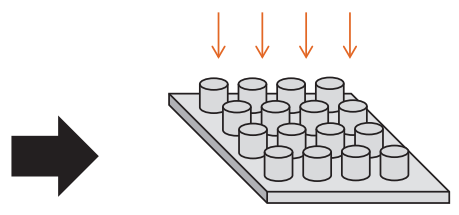

Plasma sputtering

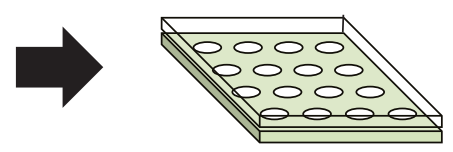

Pour the mixture into the mold

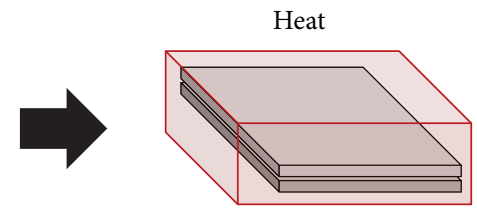

The mixture was cured in the oven $\left(80^{\circ} \mathrm{C} / 3 \mathrm{~h}\right)$
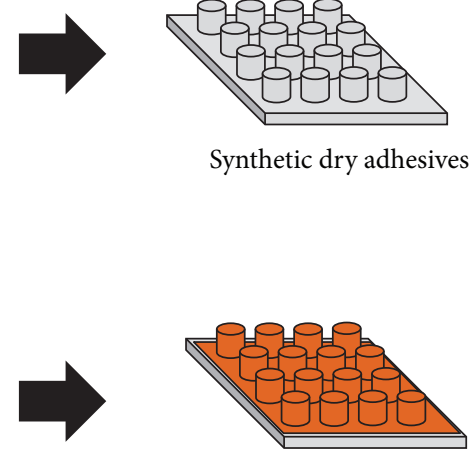

Metal coated synthetic dry adhesives

FIGURE 1: Fabrication process of synthetic dry adhesives coated with metal.

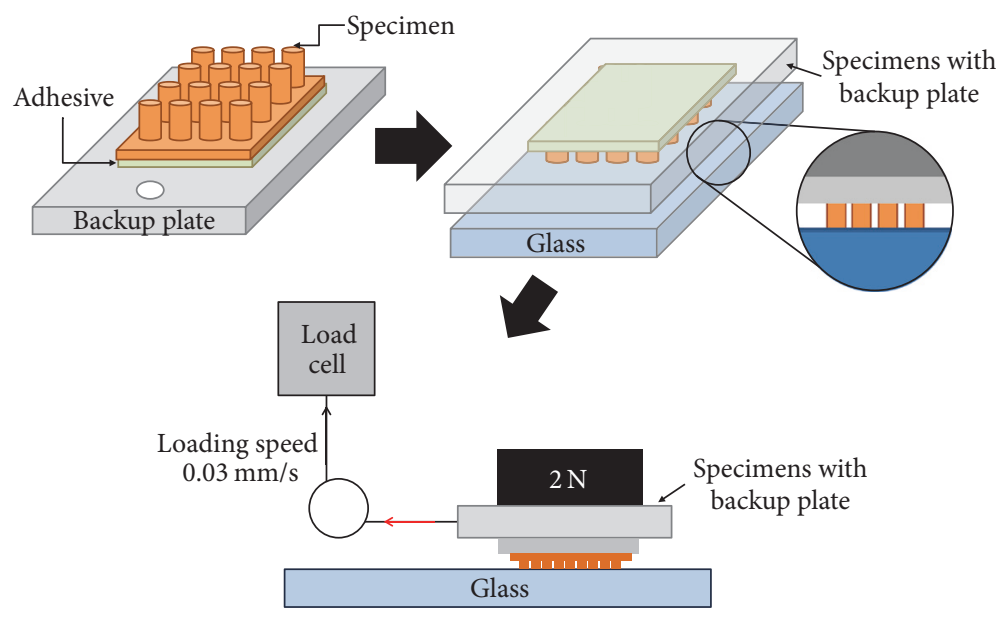

FIGURE 2: Schematic configuration of the frictional adhesion tests.

less than a $15 \%$ error when measuring the data. On the other hand, the zinc-coated synthetic dry adhesive displayed a different tendency. The shear adhesion strength increased monotonically from $5.5 \mathrm{kPa}$ to $9.6 \mathrm{kPa}$ and then decreased to $8.5 \mathrm{kPa}$. It reached maximum at $9.6 \mathrm{kPa}$ when the zinc layer thickness was $8 \mathrm{~nm}$, and the maximum error was 9.6\%. SDAs coated with gold had a relatively low shear adhesion strength compared to the other two metals. The shear adhesion strength varied from $3.6 \mathrm{kPa}$ to $6.3 \mathrm{kPa}$ with a maximum error of $12.3 \%$ in the thickness range from $2 \mathrm{~nm}$ to $10 \mathrm{~nm}$. The 


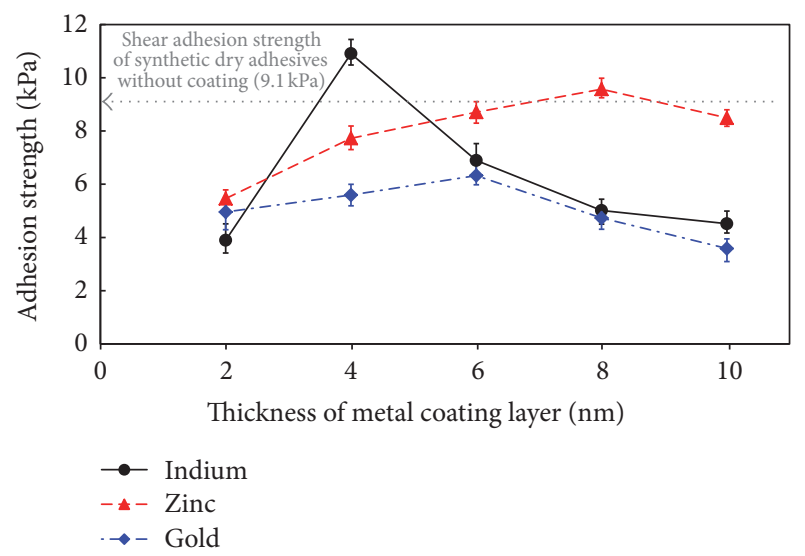

FIGURE 3: Shear adhesion strength of synthetic dry adhesives with respect to coating materials and thickness (the gray dotted line indicates the shear adhesion strength of synthetic dry adhesives without a metal coating).

maximum strength was $6.3 \mathrm{kPa}$ when the layer of gold was $6 \mathrm{~nm}$.

The adhesion characteristics of the SDAs depend on the Van der Waals forces [5]. The Van der Waals force (Fvdw) between the two flat surfaces can be calculated as follows [47]:

$$
F=-\frac{A}{\left(6 \pi D^{2}\right)},
$$

where $A$ and $D$ are the Hamaker constant $(J)$ and cut-off distance $(\approx 0.165 \mathrm{~nm})$. A can also be derived from the surface energy as follows [46]:

$$
A=24 \pi D^{2} \gamma=24 \pi D^{2} \sqrt{\gamma_{1} \gamma_{2}}
$$

where $\gamma_{1}$ and $\gamma_{2}$ represent the surface energy of the contact materials (dyne $/ \mathrm{cm})$, respectively. Therefore, the adhesion characteristics of the SDAs can improve if the SDAs are fabricated using a material with a high surface energy.

On the other hand, the Van der Waals forces are proportional to bending the SDAs due to an increase in the contact area [15], and bending is inversely proportional to the effective stiffness of the SDAs. This means that the increase in stiffness might lead to a reduction in the adhesion characteristics of the SDAs.

Table 3 shows the calculated bending stiffness of the SDAs with and without metal coatings as well as the surface energy of the coating metals. The surface energies of the coating metals are much higher than those of PDMS (about 20 to 70 times), but the bending stiffness of the SDAs increased by coating metals on the PDMS (about 10 100 times).

Two different results were reported regarding the effect of the stiffness of the SDAs [22]. Regarding the material properties, SDAs made with a hard polyurethane acrylate (PUA) with $320 \mathrm{MPa}$ of modulus had a higher adhesion strength compared to those with a soft PUA of $19.8 \mathrm{MPa}$. However, from the point of view of the structural stiffness, SDAs made with a soft PUA with a high effective stiffness had a lower adhesion strength compared to those with a low effective stiffness.
The results calculated in Table 3 and the reported results [22] indicate that different materials have different adhesion characteristics, and only the modulus or structural stiffness is not unique factor in determining the adhesion characteristics of the SDAs. Whether the effects of the surface energy or stiffness of SDAs are more important to the adhesion characteristics has not been reported, so we could not estimate a change in the adhesion strength by the metal coatings. However, the adhesion strength of the SDAs with metal coatings might be higher or lower compared to those without metal coatings.

Based on the maximum shear adhesion strength, SDAs coated with indium and zinc had a higher strength compared to those without coatings, but the gold-coated ones had a relatively low adhesion strength. From the results of the shear adhesion test, we selected the optimal thickness of the layer for each metal: $4 \mathrm{~nm}, 8 \mathrm{~nm}$, and $6 \mathrm{~nm}$ for indium, zinc, and gold, respectively.

3.2. Durability of the SDAs. The durability of the SDAs was evaluated with and without metal coating by repeating frictional adhesion tests. The changes in the shear adhesion strength for each metal are shown in Figure 4.

The reference durability data of the SDAs without a coating is shown in Figure 4(a). The shear adhesion strength decreased drastically from $9.1 \mathrm{kPa}$ to $4.1 \mathrm{kPa}$ during the first 50 repetitions. This displays reductions in the strength of $54.3 \%$ and a reduction rate of $0.099 \mathrm{kPa} /$ repetition. Until 90 repetitions, a relatively gradual reduction in the shear adhesion strength was observed, and the reduction rate was $0.023 \mathrm{kPa} /$ repetition. After 90 repetitions, the shear adhesion strength decreased monotonically with a reduction rate of $0.034 \mathrm{kPa} /$ repetition and then gradually reduced to $1.3 \mathrm{kPa}$ at 200 repetitions with a reduction rate of $0.011 \mathrm{kPa} /$ repetition. After 200 repetitions, only $14 \%$ of initial shear adhesion strength was retained.

Indium-coated SDAs had the highest initial shear adhesion strength, and their strength reduction was only $51.7 \%$ from $10.9 \mathrm{kPa}$ to $5.3 \mathrm{kPa}$ (reduction rate of $0.061 \mathrm{kPa} /$ repetition) during the first 90 repeated tests (Figure 4(b)). In the range from 100 to 180 repetitions, the reduction rate decreased as $0.021 \mathrm{kPa} /$ repetition, and then the shear adhesion strength was reduced at a reduction rate of $0.064 \mathrm{kPa} /$ repetition. Finally, the shear adhesion strength became $1.8 \mathrm{kPa}(16.3 \%$ of initial strength).

A tendency for the reduction in the shear adhesion strength for SDAs coated with zinc was similar to those with indium (Figure 4(c)). First, 90 repetitions decreased the shear adhesion strength from $9.6 \mathrm{kPa}$ to $6.3 \mathrm{kPa}$, which corresponded to a reduction rate of $0.036 \mathrm{kPa} /$ repetition. The shear adhesion strength decreased slowly to $5.15 \mathrm{kPa}$ (reduction rate of $0.016 \mathrm{kPa} /$ repetition) until 160 repetitions, and then the reduction rate increased at $0.032 \mathrm{kPa} /$ repetition. In total, $36.5 \%$ of the initial shear adhesion strength $(3.5 \mathrm{kPa})$ was maintained after 200 repetitions.

As shown in Figure 4(d), SDAs with a gold coating layer had a different trend in the shear adhesion strength compared to the other 3 cases. During the first 100 repetitions, the shear adhesion strength decreased from $6.4 \mathrm{kPa}$ to $2.1 \mathrm{kPa}$ at a reduction rate of $0.042 \mathrm{kPa} /$ repetition. Even though the 


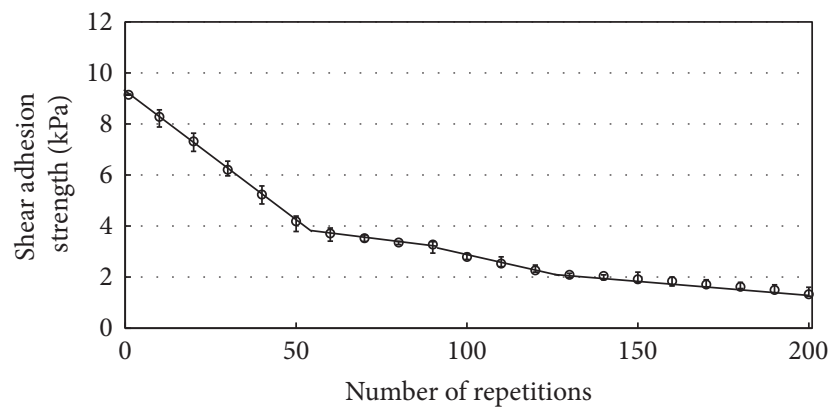

(a)

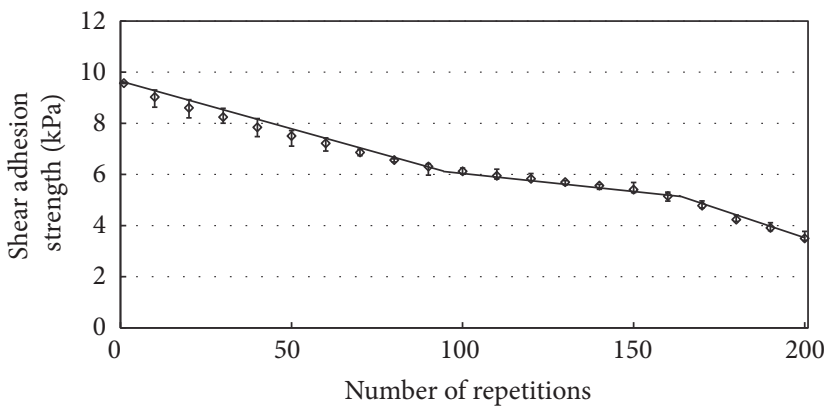

(c)

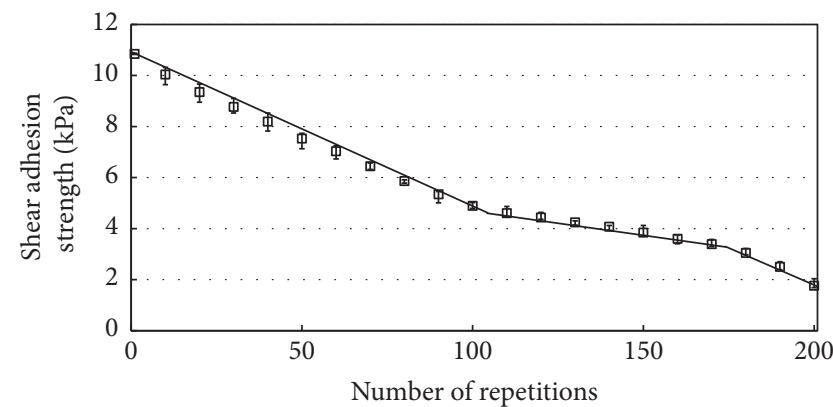

(b)

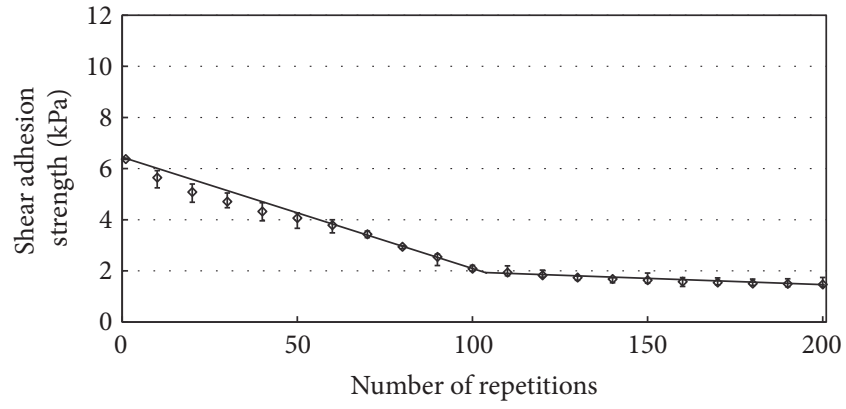

(d)

FIGURE 4: Changes in shear adhesion strength of synthetic dry adhesives (a) without coating and coated with (b) indium, (c) zinc, and (d) gold with respect to repetitions of frictional adhesion test.

TABLE 3: Comparison of the bending stiffness of SDAs with and without metal coatings and the surface energy of the coating metals.

\begin{tabular}{lcccc}
\hline & $\begin{array}{c}\text { Stiffness } \\
(\mathrm{GPa})\end{array}$ & $\begin{array}{c}\text { Coating thickness } \\
(\mathrm{nm})\end{array}$ & $\begin{array}{c}\text { Bending stiffness } \\
\left(\mathrm{Nm}^{2}\right)\end{array}$ & $\begin{array}{c}\text { Surface energy } \\
(\mathrm{mN} / \mathrm{m})\end{array}$ \\
\hline Indium & 12.7 & 4 & $3.22 \times 10^{-13}$ & 560 \\
Zinc & 96.5 & 8 & $4.14 \times 10^{-12}$ & 989 \\
Gold & 77 & 6 & $2.50 \times 10^{-12}$ & 1700 \\
PDMS & 0.00132 & - & $5.25 \times 10^{-14}$ & 23 \\
\hline
\end{tabular}

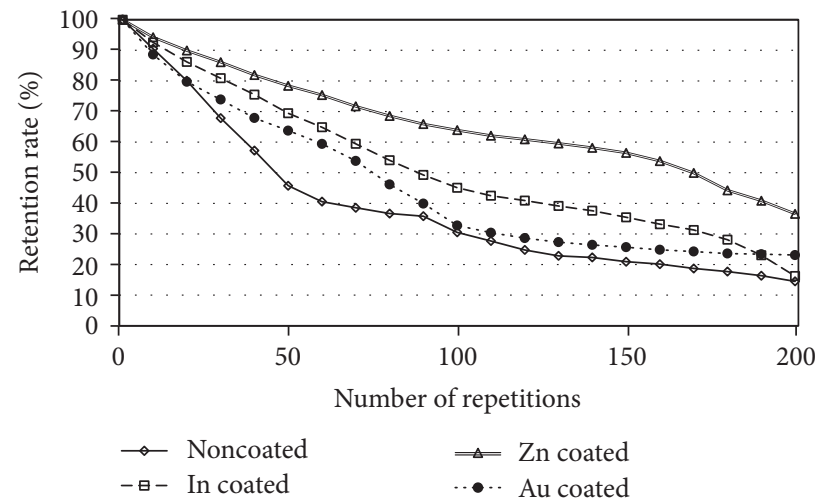

FIGURE 5: Retention rates of shear adhesion strength of synthetic dry adhesives with and without metal coatings.

reduction rate seemed to be lower than or similar to that of the other cases, the cause may be due to a relatively low initial shear adhesion strength rather than good durability. At 100 repetitions, the shear adhesion strength was only
$2.1 \mathrm{kPa}$, which is close to those of reference data after 200 repetitions. Until 200 repetitions, the reduction rate was quite low at $0.005 \mathrm{kPa} /$ repetition, but the shear adhesion strength eventually became $1.5 \mathrm{kPa}$ ( $23 \%$ of initial shear strength).

We calculated the retention rate, percentage ratio of the shear adhesion strength after repeated tests to the initial strength to compare the durability of the SDAs with and without metal coating layers based on Figure 4. As shown in Figure 5, the retention rate of the reference synthetic dry adhesive degraded the fastest, and those with metal coating layers had a relatively higher retention rate. In particular, the zinc-coated SDAs had the highest retention rate $(63.9 \%$ at 100 repetitions and $36.6 \%$ at 200 repetitions) as well as the highest shear adhesion strength $(3.5 \mathrm{kPa})$ at 200 repetitions although initial shear adhesion strength $(9.6 \mathrm{kPa})$ was lower than those of indium-coated ones $(10.8 \mathrm{kPa})$.

3.3. Surface and Structural Integrity of SDAs. The changes in the surface and structural integrity of the SDAs with and without metal coatings were investigated, as shown in Figure 6. 

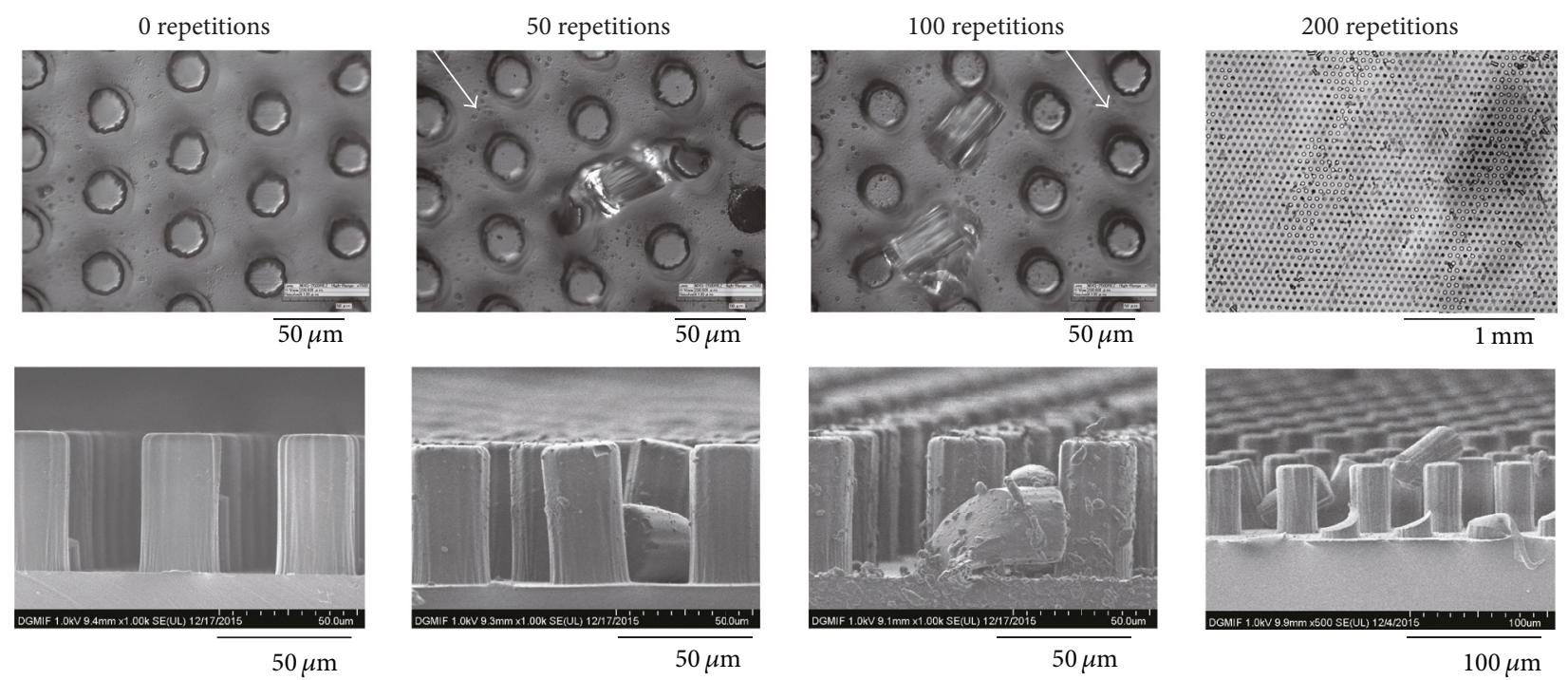

(a)
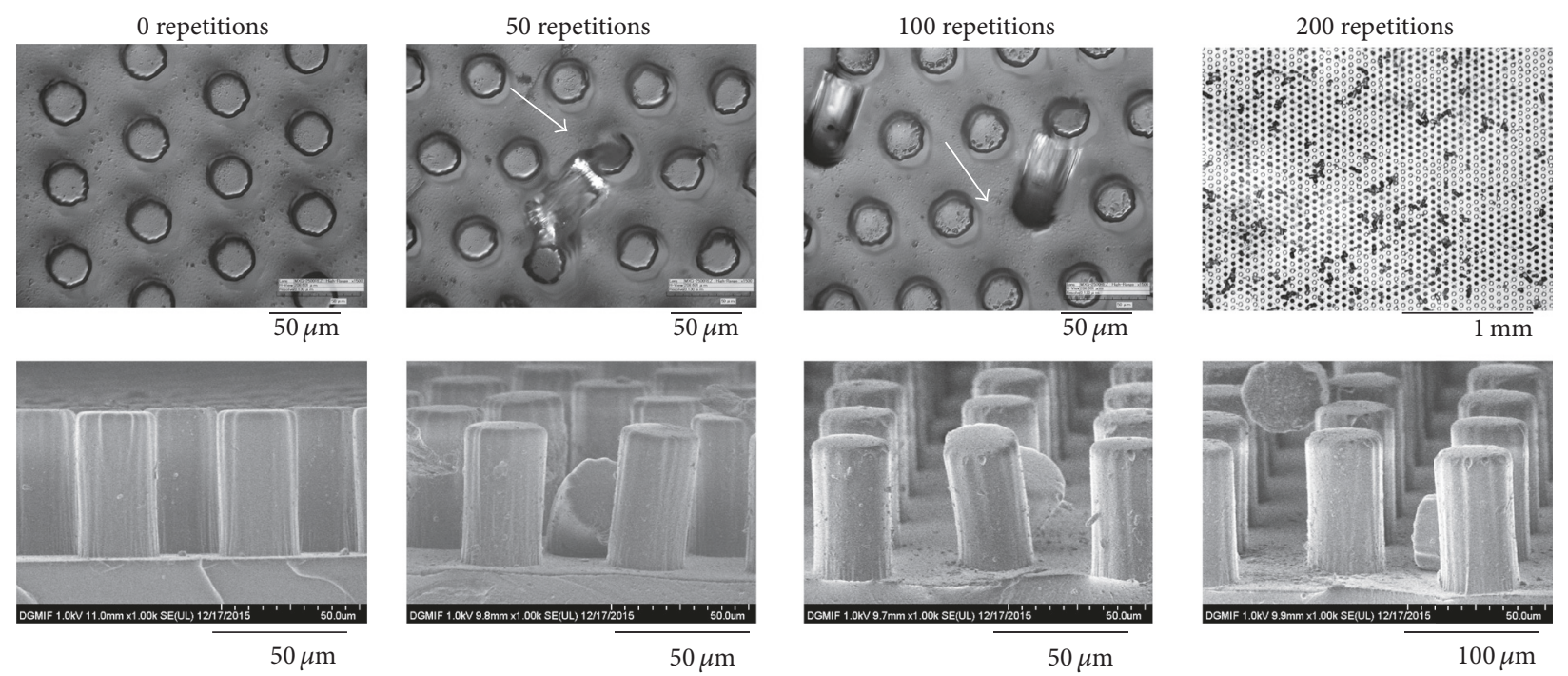

(b)
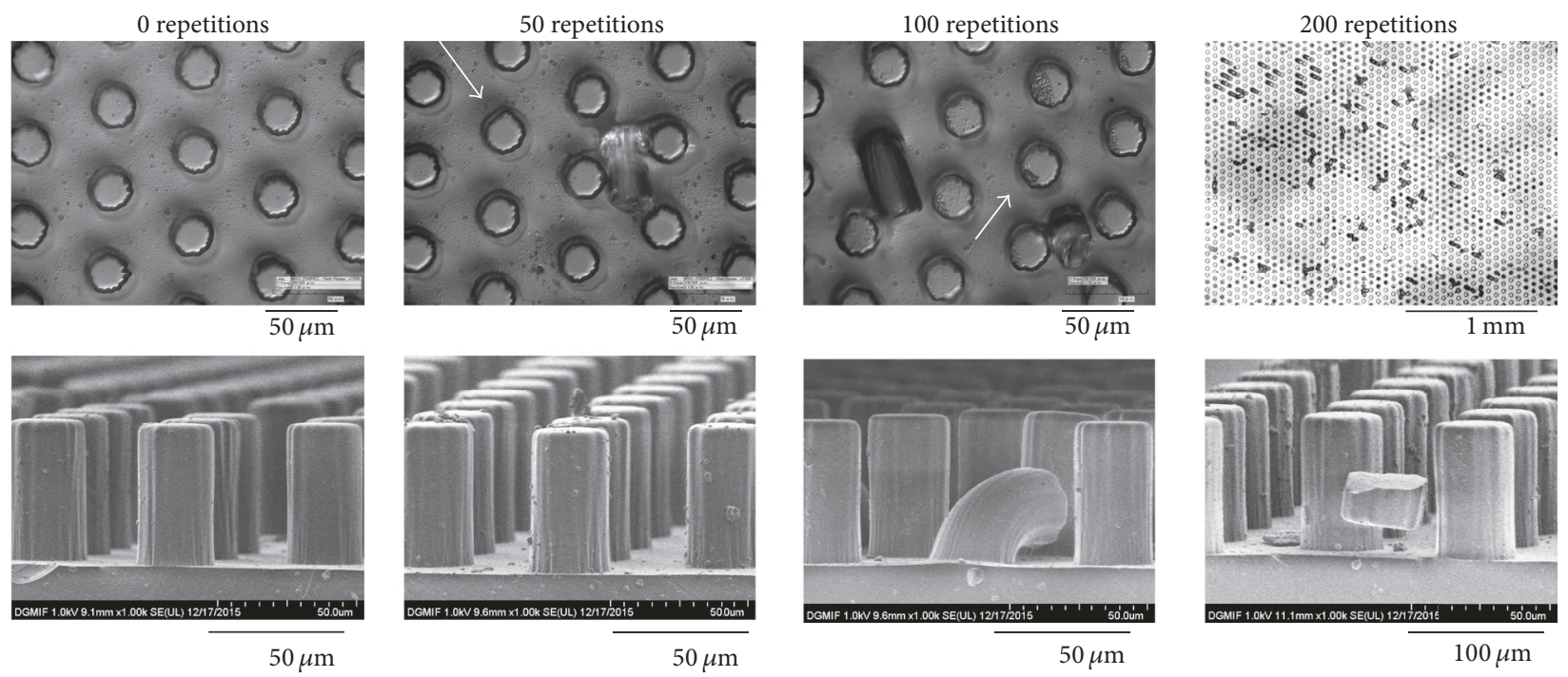

(c)

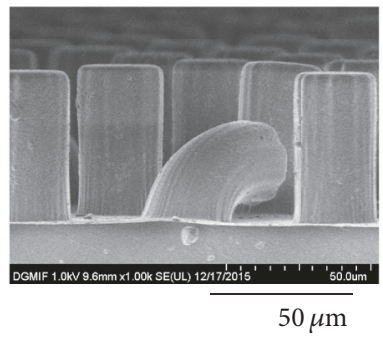

Figure 6: Continued. 


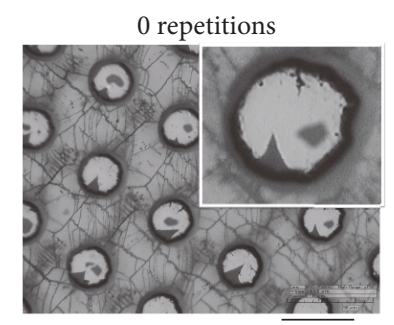

$50 \mu \mathrm{m}$

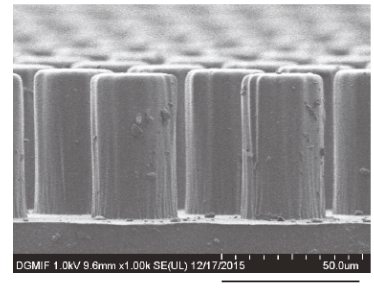

$50 \mu \mathrm{m}$

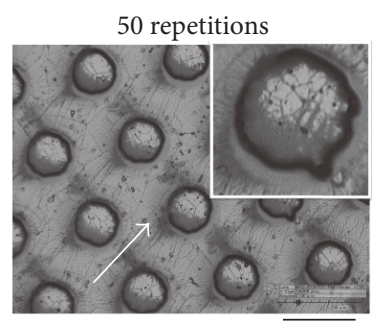

$50 \mu \mathrm{m}$

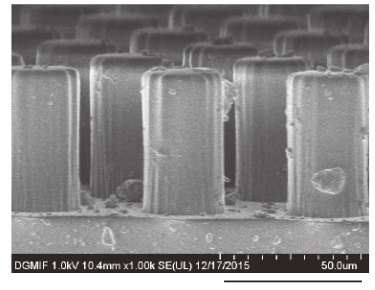

$50 \mu \mathrm{m}$

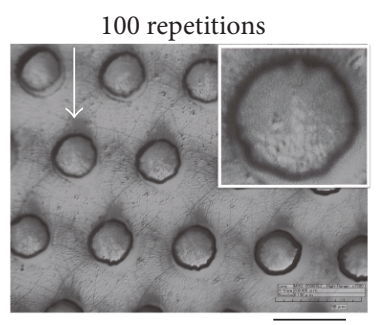

$50 \mu \mathrm{m}$

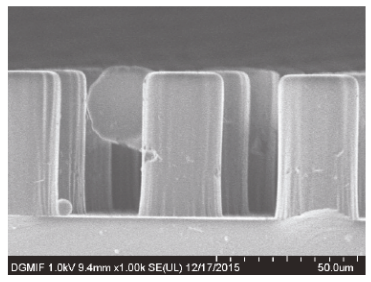

$50 \mu \mathrm{m}$
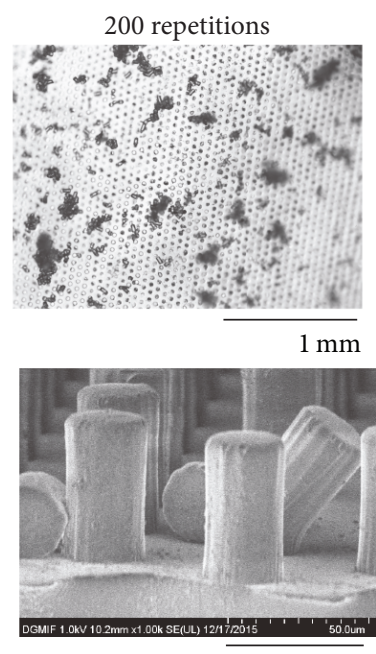

$100 \mu \mathrm{m}$

(d)

FIGURE 6: Changes of surface and structural integrity of SDAs (a) without metal coatings and with (b) indium coating, (c) zinc coating, and (d) gold coating (the inset shows the enlarged view of wear sites).

We could observe a large amount of wear on the top surfaces of the reference SDAs along the direction of the slide, as shown in Figure 6(a) (see the dark areas pointed by the arrows) after only the first 50 repetitions. The fracture on the root of the micropatterns appeared in some places at 50 repetitions because the top surface wear made a larger deformation, even though the wear was not severe until 100 repetitions. However, the fracture began to spread to the entire region after 100 repetitions and continued until 200 repetitions. At the end, $98 \%$ of the micropatterns were fractured.

Indium-coated SDAs kept the top surfaces of micropatterns relatively less worn than those of the reference until 50 repetitions due to the metal coating layer, as shown in Figure 6(b). From 50 to 100 repetitions, the wear on the top surfaces increased. At 100 repetitions, we could observe selfadhesion due to large bending deformation and some fractures at the middle of micropillars rather than the root in the reference. After 200 repetitions, fractures occurred on both the middle and root of the micropillars, and debris formed between the unfractured micropillars. At 200 repetitions, $47 \%$ of the micropatterns were in self-adhesion or large bending, and $15 \%$ were fractured.

Figure 6(c) presents the surfaces of SDAs coated with zinc. Unlike the above two cases, small abrasive particles of the zinc layer were attached on the micropillars at 50 repetitions. This means that the wear tendency of the zinc-coated SDAs is abrasion, but the above two cases represent plastic deformation, which led to the contact area becoming wider. However, the degree of wear was not severe compared to the other cases until 100 repetitions. After 100 repetitions, a large bending deformation was observed, and only $12 \%$ of the micropillars were fractured on the root at 200 repetitions. A tendency in the bending deformation and fractures was similar to that of the indium-coated ones.

Cracks were observed on a gold-coated back-up plate of the SDAs (see scratch-shaped lines in Figure 6(d)), and the top surfaces of the micropatterns were relatively bright due to the gold coating. After 50 repetitions, cracks appeared on the top surfaces, and the coated gold partially wore due to the repetitive contact. The gold-coated layer kept peeling until 100 repetitions, and it totally wore out on the top surfaces at 100 repetitions. From 100 to 200 repetitions, fractures in the micropatterns continued, similarly to the reference. Finally $89 \%$ of the SDAs were removed.

3.4. Discussion. An investigation of the surfaces and structural integrity of the SDAs could explain the tendency to reduce the shear adhesion strength through repeated use.

For the reference SDAs, wear on the top surfaces accelerated the degradation of the shear adhesion strength during the first 50 repetitions. A partial fracture was observed from 50 to 100 repetitions, and a total fracture observed by 200 repetitions resulted in a further reduction in the shear adhesion strength as a different trend. Fractured micropatterns occupied $98 \%$ of the entire area, which made the shear adhesion strength only $14 \%$ of the initial strength. The tendency for a reduction in strength implies that the most effective factors are the wear of the contact surfaces and the fracture of the micropatterns. Therefore, we can improve the durability of the SDAs if the contact surface is protected, wear is reduced, and strength of the micropillars increased. In this aspect, fabricating thin metal layers on the contact surfaces is an effective counterplan because the thin metal layers enhance the wear resistant contact surfaces and strengthen the micropillars by covering weak polymer materials.

The indium-coated layer could decrease the wear of the top surfaces until 100 repetitions compared to those of the reference, as shown in Figure 6(b). This leads to an increase in the retention rate of the share adhesion strength, as shown in Figure 5. However, a relatively low strength and stiffness for indium were not sufficient to decrease the bending deformation and reinforce the micropillars. After 
180 repetitions, an abrupt reduction in the shear adhesion strength was observed, as shown in Figure 4(b).

For comparison, the zinc-coated SDAs had a higher durability though a reduction in the shear adhesion strength similar to those with indium, as shown in Figure 6(c). The wear of the top surfaces was not noticeable until 100 repetitions as hardness of zinc-coated layer is much higher than that of indium, and it retained $63.9 \%$ of the initial shear adhesion. In addition, a relatively higher strength and stiffness of zinc than indium resulted in less bending deformation and more strengthening of the micropillars. A retention rate of $36.5 \%$ compared to the initial shear adhesion strength was achieved by good mechanical properties of the zinc-coated layer.

Gold-coated SDAs showed a different tendency even though the strength and stiffness of gold are the highest among three coating metals where the hardness is comparable to zinc. As shown in Figure 6(d), the main reason for the reduction of shear adhesion strength was exfoliation of goldcoated layer on SDAs. In general, the adhesion between the gold and polymers is relatively weaker than that of the other metals [48]. Therefore, peeling the gold-coated layer resulted in a relatively large drop in the shear adhesion strength. After 100 repetitions, the gold-coated SDAs had a trend close to reference since no gold layer remained.

\section{Conclusions}

We fabricated four different SDAs with and without metalcoated layers to investigate the effects of the metal coating on the durability of the shear adhesion strength. Among the coating materials that were considered, zinc-coated SDAs had not only the highest shear strength $(10.8 \mathrm{kPa})$, but also durability in repeated tests (retention rate of $63.9 \%$ at 100 repetitions and $36.5 \%$ at 200 repetitions). These results are more than double the strength of the SDAs without any coating. Surface observations with respect to the number of repetitions revealed important factors of their durability, including the mechanical properties (hardness, strength, and stiffness) of the coating metal and adhesion characteristics of the coating metals to the micropillars.

\section{Conflicts of Interest}

The authors declare that they have no conflicts of interest.

\section{Acknowledgments}

This work was supported by the National Research Foundation of Korea Grant funded by the Korean Government (NRF-2012S1A2A1A01030569) and in part by the National Research Foundation of Korea Grant funded by the Korean Government (NRF-2016R1A2B4014603).

\section{References}

[1] L. F. Boesel, C. Cremer, E. Arzt, and A. D. Campo, "Geckoinspired surfaces: A path to strong and reversible dry adhesives," Advanced Materials, vol. 22, no. 19, pp. 2125-2137, 2010.

[2] M. K. Kwak, C. Pang, H.-E. Jeong et al., "Towards the next level of bioinspired dry adhesives: New designs and applications,"
Advanced Functional Materials, vol. 21, no. 19, pp. 3606-3616, 2011.

[3] K. Autumn, Y. A. Liang, S. T. Hsieh et al., "Adhesive force of a single gecko foot-hair," Nature, vol. 405, no. 6787, pp. 681-685, 2000.

[4] K. Autumn, S. T. Hsieh, D. M. Dudek, J. Chen, C. Chitaphan, and R. J. Full, "Dynamics of geckos running vertically," Journal of Experimental Biology, vol. 209, no. 2, pp. 260-272, 2006.

[5] K. Autumn, M. Sitti, Y. A. Liang et al., "Evidence for van der Waals adhesion in gecko setae," Proceedings of the National Academy of Sciences of the United States of America, vol. 99, no. 19, pp. 12252-12256, 2002.

[6] D. Campolo, S. Jones, and R. S. Fearing, "Fabrication of gecko foot-hair like nano structures and adhesion to random rough surfaces," in Proceedings of the 2003 3rd IEEE Conference on Nanotechnology, IEEE-NANO 2003, pp. 856-859, usa, August 2003.

[7] W. R. Hansen and K. Autumn, "Evidence for self-cleaning in gecko setae," Proceedings of the National Academy of Sciences of the United States of America, vol. 102, no. 2, pp. 385-389, 2005.

[8] RB. Huey and PE. Hertz, "Effects of body size and slope on sprint speed of a lizard (Stellio(Agama) Stellio). J Exp Biol," in Hertz PE. Effects of body size and slope on sprint speed of a lizard (Stellio(Agama) Stellio). J Exp Biol, pp. 97-401, 97, 401-9, 1982.

[9] C. Greiner, A. Del Campo, and E. Arzt, "Adhesion of bioinspired micropatterned surfaces: Effects of pillar radius, aspect ratio, and preload," Langmuir, vol. 23, no. 7, pp. 3495-3502, 2007.

[10] R. Spolenak, S. Gorb, and E. Arzt, "Adhesion design maps for bio-inspired attachment systems," Acta Biomaterialia, vol. 1, no. 1, pp. 5-13, 2005.

[11] A. Del Campo, C. Greiner, I. Álvarez, and E. Arzt, "Patterned surfaces with pillars with controlled 3D tip geometry mimicking bioattachment devices," Advanced Materials, vol. 19, no. 15, pp. 1973-1977, 2007.

[12] A. Mahdavi, L. Ferreira, C. Sundback et al., "A biodegradable and biocompatible gecko-inspired tissue adhesive," Proceedings of the National Academy of Sciences of the United States of America, vol. 105, no. 7, pp. 2307-2312, 2008.

[13] H. Gao and H. Yao, "Shape insensitive optimal adhesion of nanoscale fibrillar structures," Proceedings of the National Academy of Sciences of the United States of America, vol. 101, no. 21, pp. 7851-7856, 2004.

[14] E. Arzt, S. Gorb, and R. Spolenak, "From micro to nano contacts in biological attachment devices," Proceedings of the National Academy of Sciences of the United States of America, vol. 100, no. 19, pp. 10603-10606, 2003.

[15] Y. Tian, N. Pesika, H. Zeng et al., "Adhesion and friction in gecko toe attachment and detachment," Proceedings of the National Academy of Sciences of the United States of America, vol. 103, no. 51, pp. 19320-19325, 2006.

[16] N. J. Glassmaker, A. Jagota, C.-Y. Hui, W. L. Noderer, and M. K. Chaudhury, "Biologically inspired crack trapping for enhanced adhesion," Proceedings of the National Academy of Sciences of the United States of America, vol. 104, no. 26, pp. 10786-10791, 2007.

[17] A. K. Geim, S. V. Dubonos, I. V. Grigorieva, K. S. Novoselov, A. A. Zhukov, and S. Y. Shapoval, "Microfabricated adhesive mimicking gecko foot-hair," Nature Materials, vol. 2, no. 7, pp. 461-463, 2003.

[18] S. Reddy, E. Arzt, and A. Del Campo, "Bioinspired surfaces with switchable adhesion," Advanced Materials, vol. 19, no. 22, pp. 3833-3837, 2007. 
[19] H. E. Jeong, S. H. Lee, P. Kim, and K. Y. Suh, "Stretched polymer nanohairs by nanodrawing," Nano Letters, vol. 6, no. 7, pp. 15081513, 2006.

[20] B. Aksak, M. P. Murphy, and M. Sitti, "Adhesion of biologically inspired vertical and angled polymer microfiber arrays," Langmuir, vol. 23, no. 6, pp. 3322-3332, 2007.

[21] J. Lee, R. S. Fearing, and K. Komvopoulos, "Directional adhesion of gecko-inspired angled microfiber arrays," Applied Physics Letters, vol. 93, no. 19, Article ID 191910, 2008.

[22] T.-I. Kim, H. E. Jeong, K. Y. Suh, and H. H. Lee, "Stooped nanohairs: Geometry-controllable, unidirectional, reversible, and robust Gecko-like dry adhesive," Advanced Materials, vol. 21, no. 22, pp. 2276-2281, 2009.

[23] A. del Campo and C. Greiner, "SU-8: a photoresist for highaspect-ratio and 3D submicron lithography," Journal of Micromechanics and Microengineering, vol. 17, no. 6, pp. R81-R95, 2007.

[24] M. P. Murphy, S. Kim, and M. Sitti, "Enhanced adhesion by gecko-inspired hierarchical fibrillar adhesives," ACS Applied Materials and Interfaces, vol. 1, no. 4, pp. 849-855, 2009.

[25] H. E. Jeong, J.-K. Lee, H. N. Kim, S. H. Moon, and K. Y. Suh, “A nontransferring dry adhesive with hierarchical polymer nanohairs," Proceedings of the National Academy of Sciences of the United States of America, vol. 106, no. 14, pp. 5639-5644, 2009.

[26] MP. Murphy, Biologically-inspired synthetic dry adhesives for wall-climbing robots, Doctor of Philosophy, Carnegie Mellon University, 2008.

[27] B. Yurdumakan, N. R. Raravikar, P. M. Ajayan, and A. Dhinojwala, "Synthetic gecko foot-hairs from multiwalled carbon nanotubes," Chemical Communications, no. 30, pp. 3799-3801, 2005.

[28] L. Ge, S. Sethi, L. Ci, P. M. Ajayan, and A. Dhinojwala, "Carbon nanotube-based synthetic gecko tapes," Proceedings of the National Academy of Sciences of the United States of America, vol. 104, no. 26, pp. 10792-10795, 2007.

[29] L. Qu, L. Dai, M. Stone, Z. Xia, and Z. L. Wang, "Carbon nanotube arrays with strong shear binding-on and easy normal lifting-off," Science, vol. 322, no. 5899, pp. 238-242, 2008.

[30] M. T. Northen and K. L. Turner, "A batch fabricated biomimetic dry adhesive," Nanotechnology, vol. 16, no. 8, pp. 1159-1166, 2005.

[31] T. S. Kustandi, V. D. Samper, W. S. Ng, A. S. Chong, and H. Gao, "Fabrication of a gecko-like hierarchical fibril array using a bonded porous alumina template," Journal of Micromechanics and Microengineering, vol. 17, no. 10, article no. N02, pp. N75N81, 2007.

[32] L. Qu and L. Dai, "Gecko-foot-mimetic aligned single-walled carbon nanotube dry adhesives with unique electrical and thermal properties," Advanced Materials, vol. 19, no. 22, pp. 3844-3849, 2007.

[33] B. Aksak, A design methodology for biologically inspired dry fibrillar adhesives, Doctor of Philosophy, Carnegie Mellon University, 2008.

[34] B. N. J. Persson and S. Gorb, "The effect of surface roughness on the adhesion of elastic plates with application to biological systems," Journal of Chemical Physics, vol. 119, no. 21, pp. 1143711444, 2003.

[35] K. A. Daltorio, A. D. Horchler, S. Gorb, R. E. Ritzmann, and R. D. Quinn, "A small wall-walking robot with compliant, adhesive feet," in Proceedings of the IEEE IRS/RSJ International Conference on Intelligent Robots and Systems, IROS 2005, pp. 40184023, can, August 2005.
[36] S. Kim and M. Sitti, "Biologically inspired polymer microfibers with spatulate tips as repeatable fibrillar adhesives," Applied Physics Letters, vol. 89, no. 26, Article ID 261911, 2006.

[37] S. Gorb, M. Varenberg, A. Peressadko, and J. Tuma, "Biomimetic mushroom-shaped fibrillar adhesive microstructure," Journal of the Royal Society Interface, vol. 4, no. 13, pp. 271-275, 2007.

[38] J. Davies, S. Haq, T. Hawke, and J. P. Sargent, "A practical approach to the development of a synthetic Gecko tape," International Journal of Adhesion and Adhesives, vol. 29, no. 4, pp. 380-390, 2009.

[39] D. Sameoto and C. Menon, "A low-cost, high-yield fabrication method for producing optimized biomimetic dry adhesives," Journal of Micromechanics and Microengineering, vol. 19, no. 11, Article ID 115002, 2009.

[40] D. Sameoto and C. Menon, "Deep UV patterning of acrylic masters for molding biomimetic dry adhesives," Journal of Micromechanics and Microengineering, vol. 20, no. 11, Article ID 115037, 2010.

[41] H. E. Jeong, M. K. Kwak, and K. Y. Suh, "Stretchable, adhesiontunable dry adhesive by surface wrinkling," Langmuir, vol. 26, no. 4, pp. 2223-2226, 2010.

[42] A. Parness, D. Soto, N. Esparza et al., "A microfabricated wedgeshaped adhesive array displaying gecko-like dynamic adhesion, directionality and long lifetime," Journal of the Royal Society Interface, vol. 6, no. 41, pp. 1223-1232, 2009.

[43] S. S. Chary, Microfabrication of Bio-Inspired Adhesive Systems, Doctor of Philosophy, University of California, 2013.

[44] E. Cheung, Biologically inspired fibrillar adhesion and friction on biological tissues: Modeling, Fabrication and applications [PhD thesis], Carnegie Mellon University, 2008.

[45] G. H. Kim, T. C. An, and H. Y. Hwang, "Effects of metal coatings on adhesive characteristics of gecko-like micro structures," Transactions of the Korean Society of Mechanical Engineers, A, vol. 39, no. 11, pp. 1099-1103, 2015.

[46] M. Sitti and R. S. Fearing, "Synthetic gecko foot-hair micro/ nano-structures as dry adhesives," Journal of Adhesion Science and Technology, vol. 17, no. 8, pp. 1055-1073, 2003.

[47] J. N. Israelachivili, Intermolecular and Surface Forces, Elsevier, 3rd edition, 2011.

[48] Y. Yamada, DR. Wheeler, and DH. Bcukley, "Adhesion between polymers and evaporated gold and nickel films. National Aeronautics and Space Administration," Tech. Rep., 1984. 

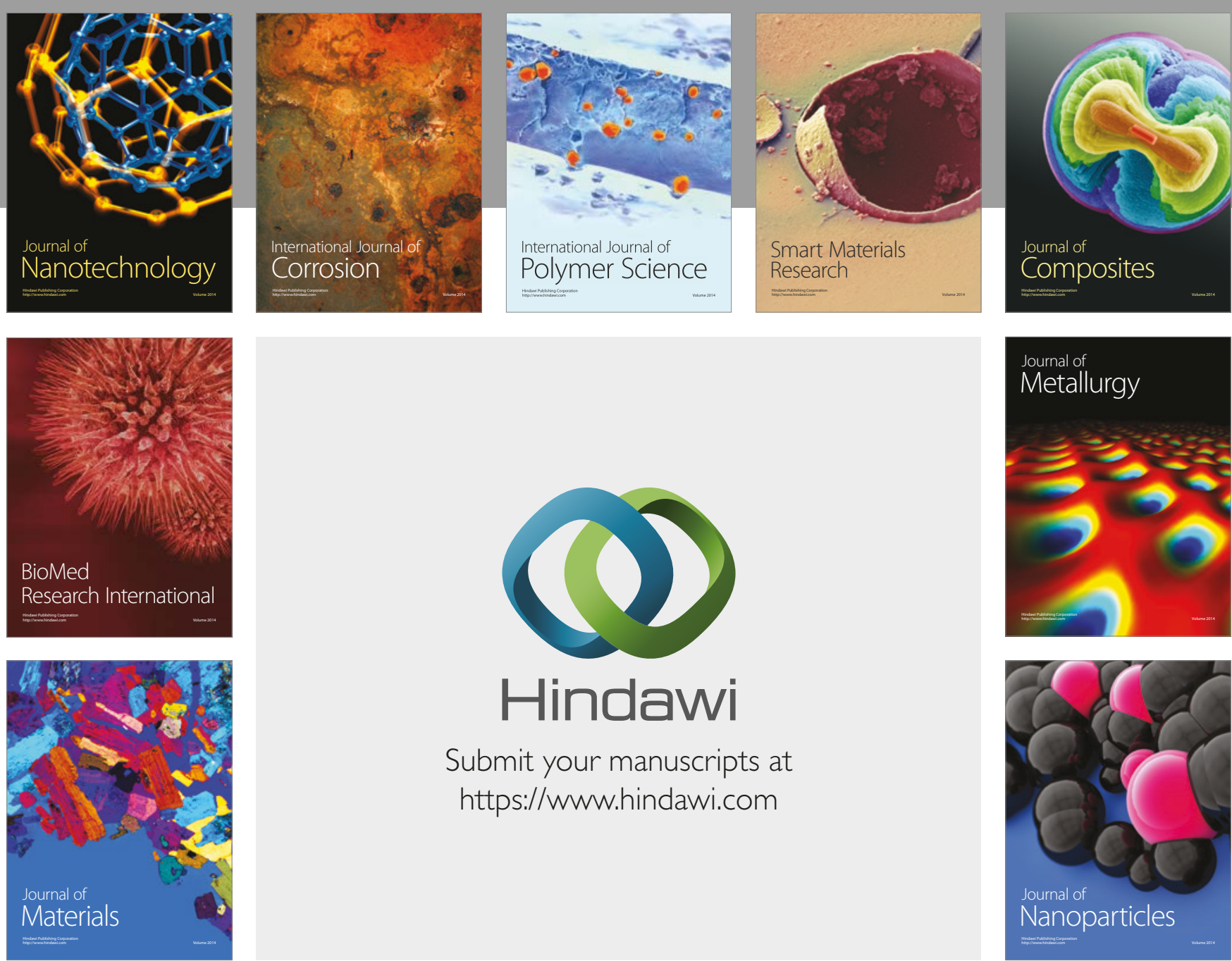

\section{Hindawi}

Submit your manuscripts at

https://www.hindawi.com
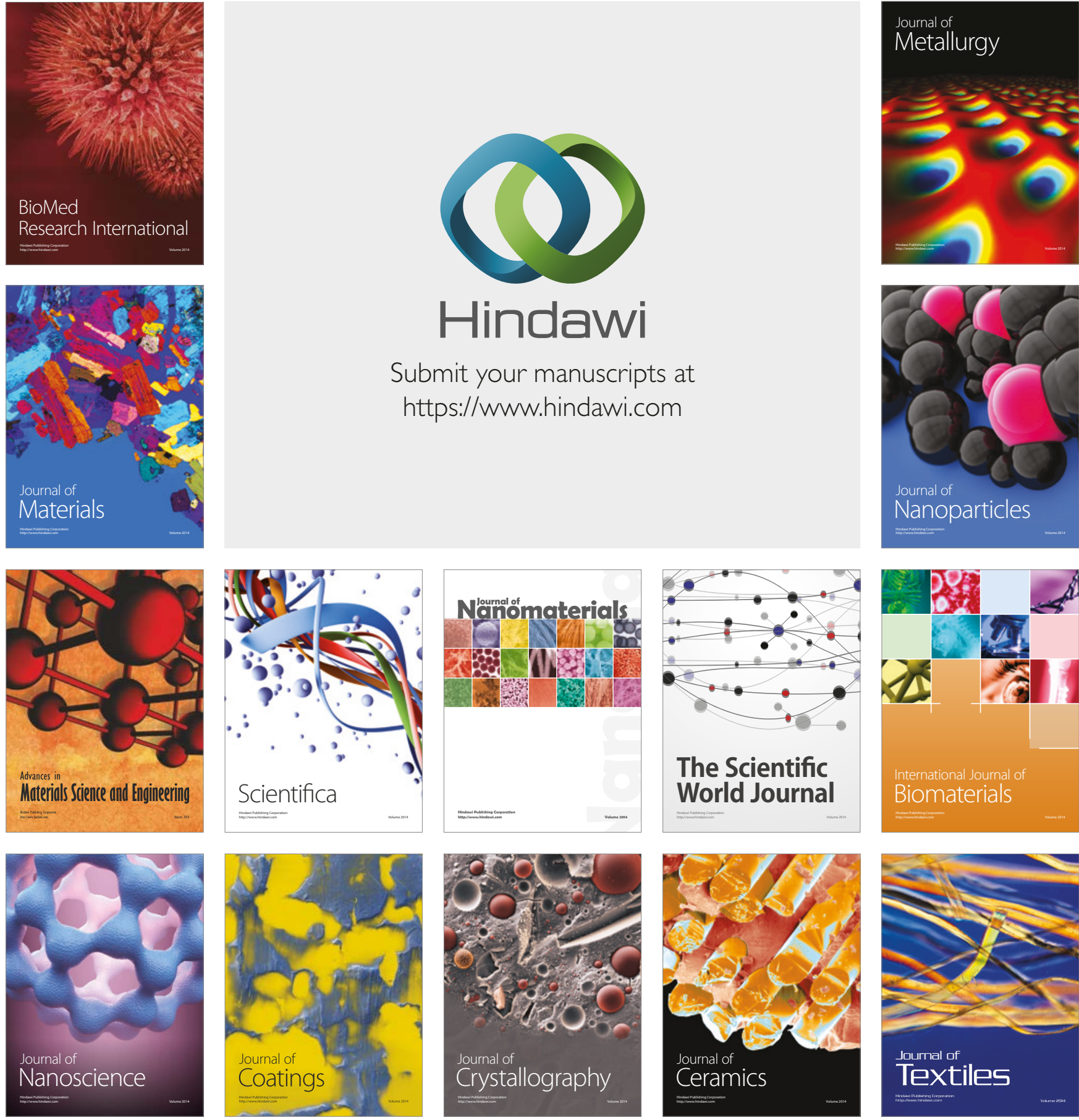

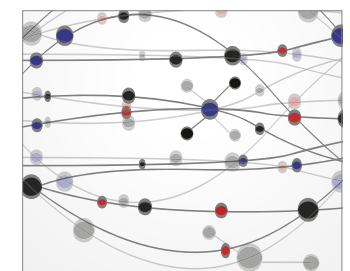

The Scientific World Journal
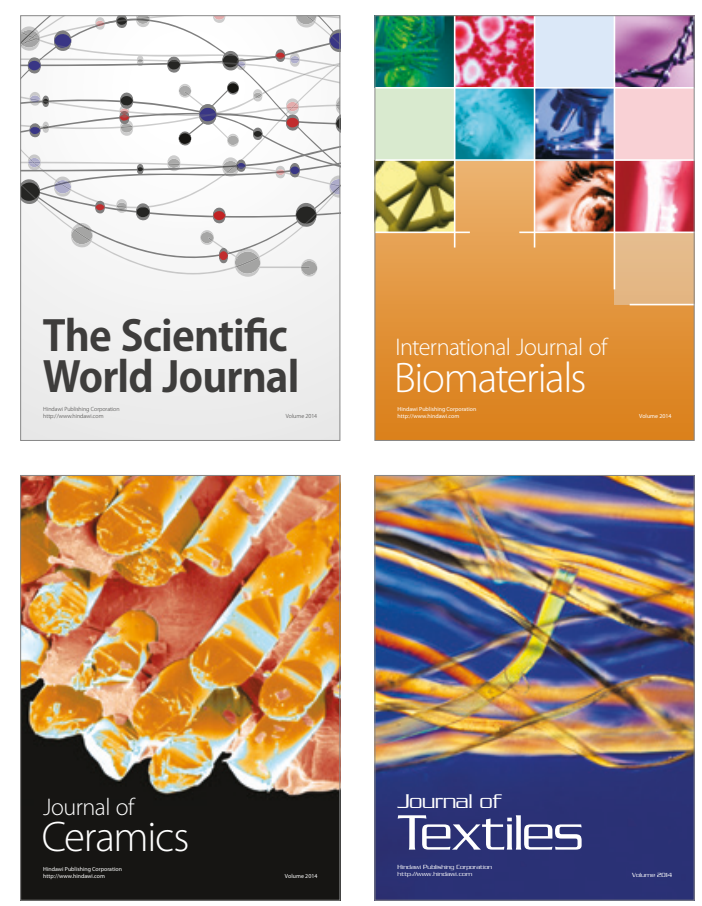